\title{
Convergence Analysis of Quantized Primal-dual Algorithm in Quadratic Network Utility Maximization Problems
}

\author{
Ehsan Nekouei, Girish Nair, Tansu Alpcan
}

\begin{abstract}
This paper examines the effect of quantized communications on the convergence behavior of the primal-dual algorithm in quadratic network utility maximization problems with linear equality constraints. In our set-up, it is assumed that the primal variables are updated by individual agents, whereas the dual variables are updated by a central entity, called system, which has access to the parameters quantifying the system-wide constraints. The notion of differential entropy power is used to establish a universal lower bound on the rate of exponential mean square convergence of the primal-dual algorithm under quantized message passing between agents and the system. The lower bound is controlled by the average aggregate data rate under the quantization, the curvature of the utility functions of agents, the number of agents and the number of constraints. An adaptive quantization scheme is proposed under which the primal-dual algorithm converges to the optimal solution despite quantized communications between agents and the system. Finally, the rate of exponential convergence of the primal-dual algorithm under the proposed quantization scheme is numerically studied.
\end{abstract}

\section{INTRODUCTION}

Allocating resources to multiple agents in a large network usually requires solving a massive optimization problem with many parameters which are not known at the system level [1]-[2], e.g., the utility functions of agents are usually considered as private information of agents, and are only known by individual agents. In the seminal paper [3], Kelly et al. proposed the network utility maximization (NUM) approach for solving resource allocation problem in large networks. This approach allows the underlying optimization problem to be solved using various distributed computation architectures, e.g., primal, dual and primal-dual decompositions. Each architecture decomposes the original optimization problem into a set of smaller sub-problems which can be locally solved by agents by exchanging only a small number of variables between themselves. The diversity of decomposition architectures allows the system designer to select the best possible decomposition method based on the network architecture and the design criteria.

In each architecture, finding the optimal solution of a NUM problem requires the exchange of different information between agents and system, e.g., pricing signals. However, due to the inherent capacity limitation of communication channels, the information exchange between network components can only be performed in the form of quantized

This work was supported by the Australian Research Council's Discovery Projects funding scheme (DP140100819).

Department of Electrical and Electronic Engineering, The University of Melbourne, VIC 3010, Australia. E-mails: \{ehsan.nekouei, gnair, tansu.alpcan\}@unimelb.edu.au message passing which can significantly degrade the performance of distributed multi-agent algorithms. The potential detrimental impact of quantization has derived intense research efforts analyzing the convergence of distributed multi-agent optimization/consensus algorithms in presence of quantized inter-agent communications.

\section{Literature Survey}

Nedić et al. [4] studied the convergence rate of an averaging-based algorithm for a cooperative multi-agent convex optimization problem in which agents collectively minimize the sum of individual objective functions, and the communication between agents is regulated by an infinitelevel, uniform quantization scheme. Rabbat et al. [5] examined the convergence rate of an incremental algorithm for solving a distributed multi-agent convex optimization problem under a uniform quantization scheme. The authors in [6] investigated the structure of the optimal quantization schemes minimizing various measures of distortion in distributed contraction-based iterative algorithms under quantized interagent communications.

In [7], the authors proposed a distributed dual averaging algorithm for solving a convex optimization problem, and studied its convergence under quantized communications. Yi and Hong in [8] proposed a zoom-in quantization scheme along with a distributed sub-gradient algorithm for solving unconstrained convex optimization problems. They showed that the proposed algorithm converges to the optimal solution of the optimization problem, and obtained lower bounds on the minimum number of required quantization levels for achieving the optimal solution. The authors in [9] studied the information flow in various distributed resource allocation algorithms using the concept of entropy power.

\section{Contributions}

This paper considers a NUM problem in which a group of agents collectively seek to maximize sum of their quadratic objective functions subject to a set of linear equality constraints. In many practical applications, e.g., communication networks, electricity grid and irrigation systems, agents only have access to their local objective functions, and the global knowledge of network-wide constraints is not available to individual agents. Instead, a central entity, hereafter called system, who has the global knowledge of system-wide constraints, is responsible to ensure that the constraints are met at all time. In this paper, we assume that the system and agents employ the primal-dual algorithm to obtain the optimal solution of the NUM problem. In the primal-dual algorithm, 
each agent updates its corresponding primal variable using the local knowledge of its objective function, whereas the dual variables are updated by the system using the global knowledge of the constraints.

At each time-step of the primal-dual algorithm, the system requires the knowledge of primal variables at previous timestep in order to update the dual variables. Similarly, agents require the values of dual variables at the previous timestep to update the primal variables at each time. Since the system and agents are not necessarily co-located, the values of primal and dual variables, at each time, are exchanged between agents and system via communication links. As the capacity of communication channels is inherently limited, due to the distance between system and agents, available transmission power and bandwidth etc, agents and system are only able to exchange quantized versions of the primal and dual variables at each time.

The quantized communication between system and agents immediately triggers the following research questions: $(i)$ How fast can the primal-dual algorithm converge to the optimal solution under a given quantization scheme?, (ii) What is the impact of communication rates between agents and system on the convergence of primal-dual algorithm? To address these questions, the current paper uses the information theoretic notion of differential entropy power to establish a universal lower bound on the rate of exponential mean square convergence of the primal-dual algorithm under a class of quantization schemes called optimum achieving (OA) quantization schemes. Under an OA quantization scheme, the primal and dual variables converge to their corresponding optimal values.

Different from previous results, which are mainly limited to uniform quantization schemes, the derived lower bound is independent of quantizer's structure, and only depends on the average aggregate data rate between agents and system. Our result indicates that, given a fixed average aggregate data rate, the quantized primal-dual algorithm can converge to the optimal solution at most exponentially fast, and the rate of exponential mean square convergence is limited by the curvature of the objective functions of agents, the average aggregate communication data rate among agents and system, number of agents and number of constraints (see Theorem 1 for more details). Also, an OA quantization scheme is proposed under which the primal-dual algorithm converges to the optimal solution. We note that the notion of differential entropy power has been used to study the impact of data rate constraints on the stability of feedback control systems, e.g., see [10] and [11].

This paper is organized as follows. Next section describes the considered NUM problem along with the quantized primal-dual update rule. Section III states our main results followed by a detailed discussion of the results. Section IV presents our numerical results for the convergence behavior of the primal-dual algorithm under quantized communications. Section V concludes the paper. All the proofs are relegated to appendices to enhance the flow of the paper.

\section{SYSTEM MODEL}

Consider a NUM problem in which a group of agents aim to cooperatively maximize the aggregate of their individual utility functions subject to a set of linear equality constraints. Let $U_{i}\left(x^{i}\right)=\frac{a_{i}}{2}\left(x^{i}\right)^{2}+c_{i} x^{i}$ be the utility function of agent $i$ where $x^{i}$ is the decision variable of agent $i, a_{i}<0$ and $c_{i} \in \mathbb{R}$. Our motivation for considering quadratic utility functions has two aspects: $(i)$ Non-linear utility functions can be approximated up to a second order by quadratic utility functions, $(\mathrm{ii})$ The analytical tractability of quadratic optimization problems provides useful insights regarding the characteristics of their optimal solutions [12].

The system and agents are interested in the solution of following optimization problem:

$$
\begin{array}{cl}
\underset{\boldsymbol{x}}{\operatorname{maximize}} & \sum_{i}^{M} U_{i}\left(x^{i}\right), \\
\text { Subject to } & A \boldsymbol{x}=\boldsymbol{b}
\end{array}
$$

where $M$ is the number of agents, $\boldsymbol{b} \in \mathbb{R}^{N}, N$ is the number of constraints, $A \in \mathbb{R}^{N \times M}$, and $\boldsymbol{x}=\left[x^{1}, \cdots, x^{M}\right]^{\top}$. The condition $N<M$ is imposed on the number of agents and the number of constraints to ensure that the feasible set of the optimization problem is non-empty. The objective function in (1) is concave, and the constraints are linear. Thus, the optimization problem (1) can be solved using standard convex optimization techniques.

Let $x_{k}^{i}$ and $\lambda_{k}^{i}$ be the primal and dual variables, respectively, under the primal-dual algorithm at time $k$. Then, the primal-dual algorithm for the NUM problem (1) can be written as

$$
\begin{aligned}
& x_{k}^{i}=x_{k-1}^{i}+\mu_{k-1}\left(a_{i} x_{k-1}^{i}+c_{i}-A_{i}^{\top} \boldsymbol{\lambda}_{k-1}\right), 1 \leq i \leq M \\
& \lambda_{k}^{j}=\lambda_{k-1}^{j}+\mu_{k-1}\left(A_{j} \boldsymbol{x}_{k-1}-b_{j}\right) \quad 1 \leq j \leq N
\end{aligned}
$$

where $\mu_{k-1}$ is the step size of the algorithm at time $k-1, b_{j}$ is the $j$ th entry of $\boldsymbol{b}, \boldsymbol{\lambda}_{k-1}=\left[\lambda_{k-1}^{1}, \cdots, \lambda_{k-1}^{N}\right]^{\top}$ and $A_{i}^{\top}$ denotes the $i$-th row of the matrix $A^{\top}$.

In our set-up, each agent only has the knowledge of its utility function and the column of matrix $A$ which corresponds to its decision variable, e.g., agent $i$ has access to the $i$ th column of matrix $A$. However, a central entity named system has the full knowledge of the matrix $A$ and vector $\boldsymbol{b}$. Thus, the dual variables are updated in each iteration by the system, whereas the primal variables are updated by agents. Let $\boldsymbol{y}_{k}$ represent the vector concatenation of $\boldsymbol{x}_{k}$ and $\boldsymbol{\lambda}_{k}$, i.e.,

$$
\boldsymbol{y}_{k}=\left[\begin{array}{l}
\boldsymbol{x}_{k} \\
\boldsymbol{\lambda}_{k}
\end{array}\right] \text {. }
$$

We assume that the vector of initial primal and dual variables, i.e., $\boldsymbol{y}_{0}$, is randomly drawn according to the probability density function $p_{\boldsymbol{y}_{0}}(\boldsymbol{y})$. This assumption allows us to analyze the convergence of the primal-dual algorithm using information theoretic tools, e.g., differential entropy and differential entropy power. In this paper, we impose the following assumptions on the step-size $\mu_{k}$ and $p_{\boldsymbol{y}_{0}}(\boldsymbol{y})$.

- $0<\mu_{k} \leq \min _{i} \frac{1}{\left|a_{i}\right|}$ for all $k$. 
- The sequence of step-sizes $\left\{\mu_{k}\right\}_{k}$ converges to $\mu^{\star}>0$ as $k$ tends to infinity.

- The distribution of initial primal and dual variables has a finite entropy. That is, $\left|-\int p_{\boldsymbol{y}_{0}}(\boldsymbol{y}) \log \left(p_{\boldsymbol{y}_{0}}(\boldsymbol{y})\right) d \boldsymbol{y}\right|<$ $\infty$.

\section{A. The Structure of Quantizer}

According to the update rule (2), at time $k$, each agent requires the knowledge of dual variables at time $k-1$ to update its decision variable. Similarly, the system requires the knowledge of primal variables at time $k-1$ in order to update the dual variables at time $k$. Since agents and system are not necessarily co-located, the values of primal and dual variables, at each time, are needed to be exchanged via communication links deployed between agents and system. However, the capacity of communication links is limited by the available transmission power, bandwidth, etc. Due to the capacity limitation of communication channels, agents and system can only exchange the quantized versions of primal and dual variables which are representable using finite number of bits.

Let $Q_{i, k}^{x}\left(x_{k}^{i}\right): \mathbb{R} \longmapsto \mathcal{A}_{i, k}^{x}$ represent the quantizer used by agent $i$ to quantize its decision variable at time $k$ where $\mathcal{A}_{i, k}^{x}$ is a finite set representing the range of $Q_{i, k}^{x}\left(x_{k}^{i}\right)$. Similarly, let $Q_{k}^{\lambda}\left(\boldsymbol{\lambda}_{k}\right): \mathbb{R}^{N} \longmapsto \mathcal{A}_{k}^{\lambda}$ represent the quantizer used by the system at time $k$ to quantize the dual variables where $\mathcal{A}_{k}^{\lambda}$ is a finite set. The quantities $\left|\mathcal{A}_{i, k}^{x}\right|$ and $\left|\mathcal{A}_{k}^{\lambda}\right|$ denote the number of quantization levels used by agent $i$ and the system, respectively, to quantize their corresponding variables at time $k$. A large value of $\left|\mathcal{A}_{i, k}^{x}\right|$ indicates that the agent $i$ transmits its primal variable with a high precision to the system whereas a small $\left|\mathcal{A}_{i, k}^{x}\right|$ indicates a poor communication channel between agent $i$ and the system. In the sequel, $Q_{k}^{\boldsymbol{x}}\left(\boldsymbol{x}_{k}\right)$ denotes the component-wise quantized version of $\boldsymbol{x}_{k}$. Also, $Q_{k}\left(\boldsymbol{y}_{k}\right)$ is used to represent the quantized version of $\boldsymbol{y}_{k}$, i.e.,

$$
Q_{k}\left(\boldsymbol{y}_{k}\right)=\left[\begin{array}{l}
Q_{k}^{\boldsymbol{x}}\left(\boldsymbol{x}_{k}\right) \\
Q_{k}^{\boldsymbol{\lambda}}\left(\boldsymbol{\lambda}_{k}\right)
\end{array}\right] .
$$

We refer to $\mathcal{Q}=\left\{Q_{k}(\cdot)\right\}_{k=0}^{\infty}$ as a quantization scheme. The average aggregate date rate per unit time under the quantization scheme $\mathcal{Q}$, i.e., $R_{\mathcal{Q}}$, is defined as [13]

$$
R_{\mathcal{Q}}=\limsup _{k \rightarrow \infty} \frac{1}{k} \sum_{t=0}^{k-1} \sum_{i=1}^{M} \log \left|\mathcal{A}_{i, t}^{\boldsymbol{x}}\right|+\log \left|\mathcal{A}_{t}^{\boldsymbol{\lambda}}\right|
$$

The primal-dual algorithm under the quantization scheme $\mathcal{Q}$ can be written as

$$
\begin{aligned}
& x_{k}^{i}=x_{k-1}^{i}+\mu_{k-1}\left(a_{i} x_{k-1}^{i}+c_{i}-A_{i}^{\top} Q_{k-1}^{\boldsymbol{\lambda}}\left(\boldsymbol{\lambda}_{k-1}\right)\right), \\
& \lambda_{k}^{j}=\lambda_{k-1}^{j}+\mu_{k-1}\left(A_{j} Q_{k-1}^{\boldsymbol{x}}\left(\boldsymbol{x}_{k-1}\right)-b_{j}\right)
\end{aligned}
$$

Let $\boldsymbol{x}^{\star}=\left[x^{1^{\star}}, \cdots, x^{M^{\star}}\right]^{\top}$ and $\boldsymbol{\lambda}^{\star}=\left[\lambda^{1^{\star}}, \cdots, \lambda^{N^{\star}}\right]^{\top}$ be the optimal values of the primal and dual variables, respectively. Also, let $\boldsymbol{y}^{\star}$ be the vector concatenation of $\boldsymbol{x}^{\star}$ and $\boldsymbol{\lambda}^{\star}$. We define $\epsilon_{k}=\boldsymbol{y}_{k}-\boldsymbol{y}^{\star}$ as the difference between the optimal solution and the primal-dual variables at time $k$.
Let $\left\|\epsilon_{k}\right\|_{2}$ denote the total distance between the values of primal-dual variables at time $k$ and their optimal values, i.e.,

$$
\left\|\boldsymbol{\epsilon}_{k}\right\|_{2}=\sqrt{\sum_{i=1}^{M}\left(x_{k}^{i}-x^{i^{\star}}\right)^{2}+\sum_{j=1}^{N}\left(\lambda_{k}^{j}-\lambda^{j^{\star}}\right)^{2}}
$$

Then, the average error norm square at time $k$ under the quantization scheme $\mathcal{Q}$ is defined as $\mathrm{E}\left[\left\|\epsilon_{\boldsymbol{k}}\right\|_{2}^{2}\right]$. Next, we define the class of optimum achieving (OA) quantization schemes.

Definition 1: The quantization scheme $\mathcal{Q}$ is called an optimum achieving (OA) quantization scheme if, under $\mathcal{Q}$, the primal and dual variables converge to their optimal values $\boldsymbol{x}^{\star}$ and $\boldsymbol{\lambda}^{\star}$. That is, $\lim _{k \rightarrow \infty} \boldsymbol{x}_{k}=\boldsymbol{x}^{\star}$ and $\lim _{k \rightarrow \infty} \boldsymbol{\lambda}_{k}=\boldsymbol{\lambda}^{\star}$. The Definition 1 implies that, under an OA quantization scheme, the quantization error does not impede the primaldual algorithm from approaching the optimal solution, and the primal-dual algorithm converges to the optimal solution of the optimization problem regardless of the quantized message passing between agents and system.

\section{Convergence Analysis}

In this section, we analyze the rate of exponential mean square convergence of the primal-dual algorithm under quantized communication between the system and agents. To this end, first, the notion of error decay exponent (EDE) is defined which captures the speed of exponential convergence of the primal-dual algorithm to the optimal solution.

Definition 2: Consider the primal-dual update rule (4) under an OA quantization scheme $\mathcal{Q}$. Then, the error decay exponent (EDE) of the primal-dual algorithm under $\mathcal{Q}$ is defined as $\liminf \operatorname{in}_{k \rightarrow \infty} \frac{1}{k} \log \mathrm{E}\left[\left\|\epsilon_{k}\right\|_{2}^{2}\right]$ where $\left\|\epsilon_{k}\right\|_{2}$ is the distance between the vector of primal-dual variables at time $k$, i.e., $\boldsymbol{y}_{k}$, and the vector of optimal solution, i.e., $\boldsymbol{y}^{\star}$.

Note that the EDE is a non-positive quantity, and a more negative EDE implies a faster convergence to the optimal solution compared with a less negative EDE. In the next theorem, we establish a universal lower bound on the EDE of primal-dual algorithm for quadratic NUM problems under quantized communication between system and agents. The proof of this theorem is based on the information-theoretic notion of entropy power, and is presented in Appendix I.

Theorem 1: Consider the primal-dual algorithm under the OA quantization scheme $\mathcal{Q}$. Let $\left\|\epsilon_{k}\right\|_{2}$ be the distance between the optimal solution and the primal-dual variables at time $k$. Then, the EDE of primal-dual algorithm under $\mathcal{Q}$ can be lower bounded as

$$
\begin{aligned}
\liminf _{k \rightarrow \infty} \frac{1}{k} \log \mathrm{E}\left[\left\|\boldsymbol{\epsilon}_{k}\right\|_{2}^{2}\right] & \\
& \geq \frac{2}{N+M}\left(\sum_{i=1}^{M} \log \left(1+\mu^{\star} a_{i}\right)-R_{\mathcal{Q}}\right) .
\end{aligned}
$$

Proof: Please see Appendix I.

Theorem 1 establishes a universal lower bound on the EDE of the primal-dual algorithm under quantized message passing between system and agents. The lower bound is universal 
in the sense that it is independent of the structure of the quantizer. Different from previous studies, which mainly focused on the uniform quantization schemes, the lower bound on the EDE in Theorem 1 does not impose any particular structure on the quantizer, and only depends on the average aggregate data rate under the quantization scheme $\mathcal{Q}$. Given an average aggregate data rate, Theorem 1 implies that the primal-dual algorithm under quantized communications converges to the optimal solution at most exponentially fast.

The lower bound in Theorem 1 is controlled by the number of agents, the number of constraints, the average aggregate data rate under the quantization scheme and the curvature of the objective functions of agents, i.e., $a_{i} \mathrm{~s}$. The lower bound in Theorem 1 decreases linearly with the average aggregate date rate per unit time, $R_{\mathcal{Q}}$, in (3). Note that, as $R_{\mathcal{Q}}$ becomes large, system and agents have more precise information about the primal and dual variables, respectively. Therefore, the primal-dual algorithm is expected to converge faster to the optimal solution as $R_{\mathcal{Q}}$ becomes large since the algorithm is updated using more accurate information at each time. Recall that the EDE is a non-positive quantity, and a more negative EDE implies a faster convergence.

The lower bound on the EDE also increases with the curvature of the objective functions of agents. Note that, as $a_{i}$ becomes less negative, the objective function of agent $i$ becomes flatter which indicates a slower convergence to the optimal solution.

\section{A. An optimum achieving quantization scheme}

In this subsection, we consider the primal-dual algorithm with a constant step-size, and we propose an optimum achieving (OA) quantization scheme which allows the primal-dual algorithm to converge to the optimal solution. The step-size is adjusted such that the primal-dual update rule under prefect communications, i.e., (2) becomes a contraction mapping with the contraction constant $\alpha \in[0,1)$. In the next section, we numerically evaluate the EDE of the primal-dual algorithm under the proposed quantization scheme. We refer to this quantization scheme as $\mathcal{Q}_{\mathrm{u}}$.

Under $\mathcal{Q}_{\mathrm{u}}$, each agent uses $R$ bits to quantize its corresponding primal variable, and the system uses $R$ bits to quantize each dual variable. At time $k=0$, agent $i$ selects the interval $I_{x_{0}^{i}}$ with length $d>0$ which contains $x_{0}^{i}$. Then, it partitions $I_{x_{0}^{i}}$ into $2^{R}$ equal length sub-intervals, and assigns a representative for each sub-interval. Finally, it transmits the representative of the sub-interval which contains $x_{0}^{i}$ to the system. Similarly, the system at time $k=0$ selects the interval $I_{\lambda_{0}^{j}}$ containing $\lambda_{0}^{j}$, and partitions it into $2^{R}$ smaller intervals, and broadcasts the representative of the sub-interval that includes $\lambda_{0}^{j}$ to agents. The length of $I_{\lambda_{0}^{j}}$ is assumed to be equal to $d$.

At time $k \geq 1$, agent $i$ and system select the intervals $I_{x_{k}^{i}}$ and $I_{\lambda_{k}^{j}}$, respectively. The intervals $I_{x_{k}^{i}}$ and $I_{\lambda_{k}^{j}}$ contain $x_{k}^{i}$ and $\lambda_{k}^{j}$, respectively, and their lengths are equal to $\alpha^{k} d$. Each interval is quantized using the quantization level $\frac{\alpha^{k} d}{2^{R}}$. Then, agents and system exchange the quantized versions of the primal and dual variables.
In the next lemma, we show that the primal-dual algorithm under $\mathcal{Q}_{\mathrm{u}}$ converges to the optimal solution.

Lemma 1: The quantization scheme $\mathcal{Q}_{\mathrm{u}}$ is an OA quantization scheme. That is, $\lim _{k \rightarrow \infty} \boldsymbol{x}_{k}=\boldsymbol{x}^{\star}$ and $\lim _{k \rightarrow \infty} \boldsymbol{\lambda}_{k}=$ $\boldsymbol{\lambda}^{\star}$ where $\boldsymbol{x}^{\star}$ and $\boldsymbol{\lambda}^{\star}$ are the vectors of optimal primal and dual variables, respectively.

Proof: Please see Appendix II.

\section{NUMERICAL RESULTS}

In this section, we numerically evaluate the convergence behavior of the primal-dual algorithm under the OA quantization scheme $\mathcal{Q}_{\mathrm{u}}$. We consider a NUM problem in which 5 agents seek to maximize the sum of their utility functions subject to 3 equality constraints. At each time, agents will quantize their primal variables using 2 bits. Also, each dual variable is quantized using 2 bits in each time-step. The parameter $d$ is set to 1 . The vector of initial primal and dual variables, i.e., $\boldsymbol{y}_{0}$, is Gaussian distributed with zero mean and covariance matrix equal to $I_{M+N}$.

Fig. 1 illustrates the trajectories of the primal and dual variables under the quantization scheme $\mathcal{Q}_{\mathrm{u}}$ for a realization of $\boldsymbol{y}_{0}$. According to Fig. 1, the primal-dual algorithm under the quantization scheme $\mathcal{Q}_{\mathrm{u}}$ converges to the optimal values of primal and dual variables. The convergence of the primaldual algorithm in Fig. 1, regardless of quantized communication between agents and system, provides a numerical confirmation for the fact that the quantization scheme $\mathcal{Q}_{\mathrm{u}}$ is an OA quantization scheme.

Fig. 2 depicts the log-mean-error-norm-square divided by $k$, i.e., $\frac{1}{k} \log \mathrm{E}\left[\left\|\boldsymbol{\epsilon}_{k}\right\|_{2}^{2}\right]$, as a function of time index $k$. For the considered NUM problem, the universal lower bound on the EDE is equal to -4.8726. According to Fig. 2, $\frac{1}{k} \log \mathrm{E}\left[\left\|\boldsymbol{\epsilon}_{k}\right\|_{2}^{2}\right]$ lies above the lower bound provided by Theorem 1 as $k$ becomes large, a behavior predicted by Theorem 1.

\section{CONCLUSION}

In this paper, we have studied the convergence rate of the primal-dual algorithm in a quadratic network utility maximization (NUM) problem in which a group of agents collectively seek to maximize the sum of their individual utility functions subject to a set of linear equality constraints. We considered a quantized communication scenario wherein the system, which is responsible for updating the dual variables, broadcasts the quantized versions of dual variables to agents, and each agent transmits the quantized version of its corresponding primal variable to the system. Using the notion of differential entropy power, a universal lower bound on the rate of exponential mean square convergence of the primal-dual algorithm was established. The lower bound is controlled by the curvature of the utility functions of agents, the average aggregate data rate under quantized message passing, number of agents and number of constraints. An 


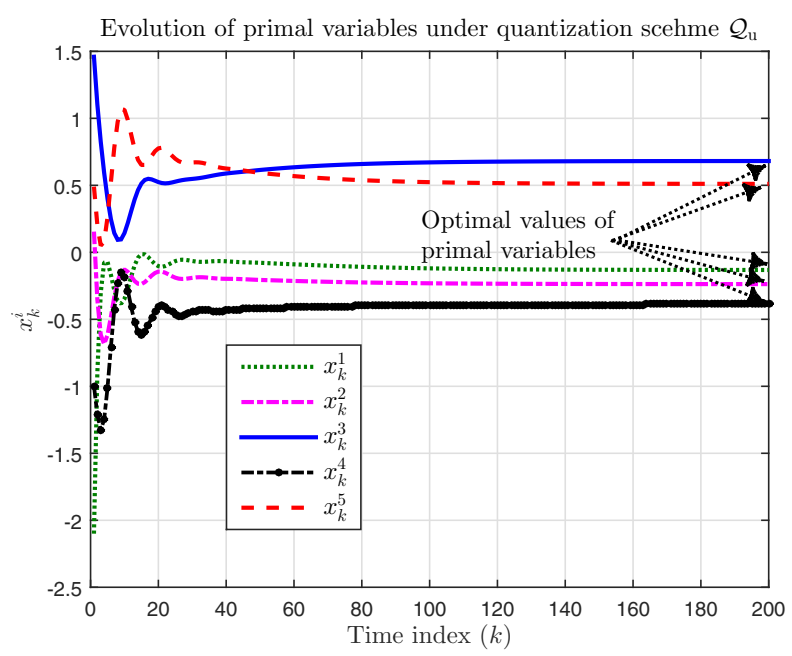

(a)

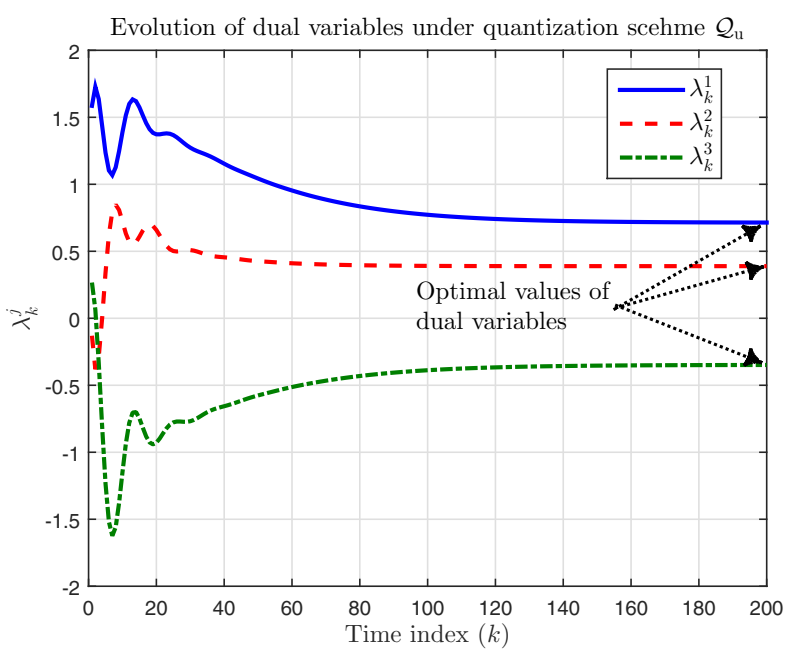

(b)

Fig. 1. Time evolution of primal variables $(a)$ and dual variables (b) under the quantization scheme $\mathcal{Q}_{\mathrm{u}}$ for a given $\boldsymbol{x}_{0}$ and $\boldsymbol{\lambda}_{0}$.

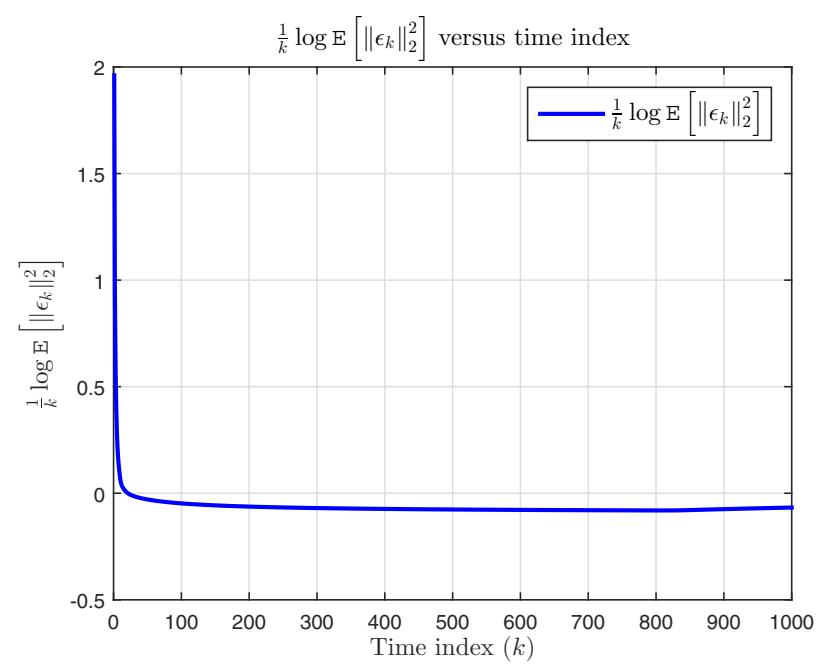

Fig. 2. log-mean-error-norm-square divided by $k$ versus time index $(k)$. adaptive quantization scheme was also proposed which allows the primal-dual algorithm to converge to the optimal solution of the NUM problem.

\section{APPENDIX I \\ PROOF OF THEOREM 1}

To prove Theorem 1, first, we define the notion of conditional differential entropy power of a random vector. Let $z \in$ $\mathbb{R}^{N+M}$ be a random vector. Then, the differential entropy power of $\boldsymbol{z}$ conditioned on the event $A=a, \mathrm{~N}[\boldsymbol{z} \mid A=a]$, is defined as $\mathrm{N}[\boldsymbol{z} \mid A=a]=\frac{1}{2 \pi \mathrm{e}} \mathrm{e}^{\frac{2}{M+N} \mathrm{~h}[\boldsymbol{z} \mid A=a]}$ where $\mathrm{h}[\boldsymbol{z} \mid A=a]$ is the conditional differential entropy of $\boldsymbol{z}$ given $A=a$. Let $\mathrm{E}_{A}[\mathrm{~N}[\boldsymbol{z} \mid A=a]]$ denote the average conditional entropy power of $\boldsymbol{z}$ given $A=a$. Then, $\mathrm{E}_{A}[\mathrm{~N}[\boldsymbol{z} \mid A=a]]$ can be upper bounded as [13]

$$
\mathrm{E}_{A}[\mathrm{~N}[\boldsymbol{z} \mid A]] \leq \mathrm{e}^{1 /(M+N)-1} \mathrm{E}\left[\|\boldsymbol{z}\|_{2}^{2}\right] .
$$

Let $\mathcal{D}_{k-1}=\left\{Q_{n}\left(\boldsymbol{y}_{n}\right)=\boldsymbol{q}_{n}\right\}_{n=0}^{k-1}$ where $\boldsymbol{q}_{n}$ is a possible output of the quantization scheme $\mathcal{Q}$ at time $i$. Then using (7), $\mathrm{E}\left[\left\|\boldsymbol{\epsilon}_{k}\right\|_{2}^{2}\right]$ can be lower bounded as

$$
\begin{aligned}
\mathrm{E}\left[\left\|\boldsymbol{\epsilon}_{k}\right\|_{2}^{2}\right] & \geq \mathrm{e}^{1-\frac{1}{M+N}} \mathrm{E}\left[\mathrm{N}\left[\boldsymbol{\epsilon}_{k} \mid \mathcal{D}_{k-1}\right]\right] \\
& \stackrel{(*)}{\geq} \frac{\mathrm{e}^{1-\frac{1}{M+N}}}{2 \pi \mathrm{e}} \mathrm{e}^{\frac{2}{M+N} \mathrm{E}\left[\mathrm{h}\left[\boldsymbol{\epsilon}_{k} \mid \mathcal{D}_{k-1}\right]\right]},
\end{aligned}
$$

where $(*)$ is obtained using the Jensen inequality. The right hand side of (8) depends on $\mathrm{h}\left[\boldsymbol{\epsilon}_{k} \mid \mathcal{D}_{k-1}\right]$ which can be simplified as

$$
\begin{aligned}
\mathrm{h}\left[\boldsymbol{\epsilon}_{k} \mid \mathcal{D}_{k-1}\right] & =\mathrm{h}\left[\boldsymbol{y}_{k}-\boldsymbol{y}^{\star} \mid \mathcal{D}_{k-1}\right] \\
& \stackrel{(*)}{=} \mathrm{h}\left[\boldsymbol{y}_{k} \mid \mathcal{D}_{k-1}\right],
\end{aligned}
$$

where $(*)$ is due to translation invariance property of differential entropy [14]. Next lemma provides an expression for $\mathrm{h}\left[\boldsymbol{y}_{n} \mid \mathcal{D}_{k-1}\right]$ in terms of $\mathrm{h}\left[\boldsymbol{y}_{n-1} \mid \mathcal{D}_{k-1}\right]$ for $n \leq k$. Later, this lemma is used to further expand $\mathrm{h}\left[\boldsymbol{y}_{k} \mid \mathcal{D}_{k-1}\right]$.

Lemma 2: For $n \leq k, \mathrm{~h}\left[\boldsymbol{y}_{n} \mid \mathcal{D}_{k-1}\right]$ can be expanded as

$\mathrm{h}\left[\boldsymbol{y}_{n} \mid \mathcal{D}_{k-1}\right]=\mathrm{h}\left[\boldsymbol{y}_{n-1} \mid \mathcal{D}_{k-1}\right]+\sum_{i=1}^{M} \log \left(1+\mu_{n-1} a_{i}\right)$.

Proof: Let $\tilde{x}_{n}^{i}=\left(1+\mu_{n} a_{i}\right) x_{n}^{i}$ and $\tilde{\boldsymbol{x}}_{n}=$ $\left[\tilde{x}_{1}^{i}, \cdots, \tilde{x}_{M}^{i}\right]^{\top}$. Let $\tilde{\boldsymbol{y}}_{n}$ be the vector concatenation of $\tilde{\boldsymbol{x}}_{n}$ and $\boldsymbol{\lambda}_{n}$. Note that $\mathrm{h}\left[\boldsymbol{y}_{n} \mid \mathcal{D}_{k-1}\right]$ can be written as

$$
\begin{aligned}
\mathrm{h}\left[\boldsymbol{y}_{n} \mid \mathcal{D}_{k-1}\right] & =\mathrm{h}\left[\boldsymbol{x}_{n}, \boldsymbol{\lambda}_{n} \mid \mathcal{D}_{k-1}\right] \\
& =\mathrm{h}\left[\tilde{\boldsymbol{x}}_{n-1}, \boldsymbol{\lambda}_{n-1} \mid \mathcal{D}_{k-1}\right] \\
& =\mathrm{h}\left[\tilde{\boldsymbol{y}}_{n-1} \mid \mathcal{D}_{k-1}\right]
\end{aligned}
$$

Note that $\tilde{\boldsymbol{y}}_{n-1}$ can be written as

$\tilde{\boldsymbol{y}}_{n-1}=\operatorname{Diag}\left(1+\mu_{n-1} a_{1}, \cdots, 1+\mu_{n-1} a_{M}, \mathbf{1}_{N}\right) \boldsymbol{y}_{n-1}$,

where $\operatorname{Diag}(\boldsymbol{z})$ is a diagonal matrix with the $i$ th diagonal element equal to $z_{i}$ and $\mathbf{1}_{N}$ is an $N$ dimensional vector of all ones. Thus,

$\mathrm{h}\left[\tilde{\boldsymbol{y}}_{n-1} \mid \mathcal{D}_{k-1}\right]=\sum_{i=1}^{M} \log \left(1+\mu_{n-1} a_{i}\right)+\mathrm{h}\left[\boldsymbol{y}_{n-1} \mid \mathcal{D}_{k-1}\right]$ 
where the equality follows from the fact that $\mathrm{h}[\boldsymbol{T} \boldsymbol{z}]=$ $\log |\operatorname{det}(T)|+\mathrm{h}[\boldsymbol{z}]$ where $\boldsymbol{T}$ is an invertible matrix.

Using Lemma $2, \mathrm{~h}\left[\boldsymbol{y}_{k} \mid \mathcal{D}_{k-1}\right]$ can be written as

$$
\mathrm{h}\left[\boldsymbol{y}_{k} \mid \mathcal{D}_{k-1}\right]=\mathrm{h}\left[\boldsymbol{y}_{0} \mid \mathcal{D}_{k-1}\right]+\sum_{j=1}^{M} \sum_{n=0}^{k-1} \log \left(1+\mu_{n} a_{j}\right) \text {. }
$$

Using (11), $\mathrm{E}\left[\mathrm{h}\left[\boldsymbol{y}_{k} \mid \mathcal{D}_{k-1}\right]\right]$ can be written as

$\mathrm{E}\left[\mathrm{h}\left[\boldsymbol{y}_{k} \mid \mathcal{D}_{k-1}\right]\right]=\sum_{j=1}^{M} \sum_{n=0}^{k-1} \log \left(1+\mu_{n} a_{j}\right)+\mathrm{E}\left[\mathrm{h}\left[\boldsymbol{y}_{0} \mid \mathcal{D}_{k-1}\right]\right]$.

Next, we use the following lemma from [13] to establish a lower bound on $\mathrm{E}\left[\mathrm{h}\left[\boldsymbol{y}_{k} \mid \mathcal{D}_{k-1}\right]\right]$.

Lemma 3: The average conditional entropy of $\boldsymbol{y}_{0}$ given $\mathcal{D}_{k-1}$, i.e., $\mathrm{E}\left[\mathrm{h}\left[\boldsymbol{y}_{0} \mid \mathcal{D}_{k-1}\right]\right]$, can be lower bounded as

$$
\mathrm{E}\left[\mathrm{h}\left[\boldsymbol{y}_{0} \mid \mathcal{D}_{k-1}\right]\right] \geq \mathrm{h}\left[\boldsymbol{y}_{0}\right]-
$$

$$
\sum_{t=0}^{k-1}\left(\sum_{i=1}^{M}\left(\log \left|\mathcal{A}_{i, t}^{\boldsymbol{x}}\right|\right)+\log \left|\mathcal{A}_{t}^{\boldsymbol{\lambda}}\right|\right) .
$$

Applying Lemma 3 to (12), we have

$$
\begin{aligned}
\mathrm{E}\left[\mathrm{h}\left[\boldsymbol{y}_{k} \mid \mathcal{D}_{k-1}\right]\right] \geq \sum_{j=1}^{M} \sum_{n=0}^{k-1} \log \left(1+\mu_{n} a_{j}\right)+\mathrm{h}\left[\boldsymbol{y}_{0}\right] \\
-\sum_{t=0}^{k-1}\left(\left(\sum_{i=1}^{M} \log \left|\mathcal{A}_{i, t}^{\boldsymbol{x}}\right|\right)+\log \left|\mathcal{A}_{t}^{\boldsymbol{\lambda}}\right|\right)
\end{aligned}
$$

Using (8), (9), (13) and the fact that $p_{\boldsymbol{y}_{0}}(\boldsymbol{y})$ has finite entropy, the EDE can be lower bounded as

$$
\begin{aligned}
& \liminf _{k \longrightarrow \infty} \frac{1}{k} \log \mathrm{E}\left[\left\|\boldsymbol{\epsilon}_{k}\right\|_{2}^{2}\right] \geq \\
& \frac{2}{M+N}\left(\liminf _{k \rightarrow \infty} \sum_{j=1}^{M} \frac{1}{k} \sum_{n=0}^{k-1} \log \left(1+\mu_{n} a_{j}\right)-R_{\mathcal{Q}}\right) .
\end{aligned}
$$

Using the fact that $\mu_{n}$ converges to $\mu^{\star}$ as $n$ tends to infinity, it can be easily shown that

$$
\begin{aligned}
\liminf _{k \rightarrow \infty} & \frac{1}{k} \log \mathrm{E}\left[\left\|\boldsymbol{\epsilon}_{k}\right\|_{2}^{2}\right] \\
& \geq \frac{2}{N+M}\left(\sum_{i=1}^{M} \log \left(1+\mu^{\star} a_{i}\right)-R_{\mathcal{Q}}\right),
\end{aligned}
$$

which completes the proof.

\section{APPENDIX II \\ PROOF OF LEMMA 1}

The primal-dual update rule under quantization can be written as

$$
\begin{aligned}
& x_{k}^{i}=x_{k-1}^{i}+\mu\left(a_{i} x_{k-1}^{i}+c_{i}-A_{i}^{\top} \boldsymbol{\lambda}_{k-1}\right)+\mu A_{i}^{\top} \boldsymbol{\delta}_{k-1}^{\boldsymbol{\lambda}} \\
& \lambda_{k}^{j}=\lambda_{k-1}^{j}+\mu\left(A_{j} \boldsymbol{x}_{k-1}-b_{j}\right)-\mu A_{j} \boldsymbol{\delta}_{k-1}^{\boldsymbol{x}}
\end{aligned}
$$

where $\boldsymbol{\delta}_{k-1}^{\boldsymbol{\lambda}}=\boldsymbol{\lambda}_{k-1}-Q_{k-1}^{\boldsymbol{\lambda}}\left(\boldsymbol{\lambda}_{k-1}\right)$ and $\boldsymbol{\delta}_{k-1}^{\boldsymbol{x}}=$ $\left(\boldsymbol{x}_{k-1}-Q_{k-1}^{\boldsymbol{x}}\left(\boldsymbol{x}_{k-1}\right)\right)$ denote the quantization error vector for dual and primal variables, respectively, at time $k-1$.
Recall that $\mu$ is selected such that the primal-dual algorithm under perfect communications becomes a contraction mapping. Using this fact and equation (16), $\left\|\epsilon_{k}\right\|_{2}$ for $k \geq 1$ can be upper bounded as

$$
\begin{aligned}
\left\|\epsilon_{k}\right\|_{2} & \leq \alpha\left\|\epsilon_{k-1}\right\|_{2}+\mu\left\|\left[\begin{array}{cc}
\mathbf{0} & A^{\top} \\
-A & \mathbf{0}
\end{array}\right]\right\|_{2}\left\|\left[\begin{array}{c}
\boldsymbol{\delta}_{k-1}^{\boldsymbol{x}} \\
\boldsymbol{\delta}_{k-1}^{\boldsymbol{\lambda}}
\end{array}\right]\right\|_{2} \\
& \stackrel{(*)}{\leq} \alpha\left\|\epsilon_{k-1}\right\|_{2}+\mu\left\|\left[\begin{array}{cc}
\mathbf{0} & A^{\top} \\
-A & \mathbf{0}
\end{array}\right]\right\|_{2} \sqrt{N+M} \frac{\alpha^{k-1} d}{2^{R}}
\end{aligned}
$$

where $(*)$ follows from the fact that the absolute value of quantization error for primal/dual variables at time $k-1$ is upper bounded by $\frac{\alpha^{k-1} d}{2^{R}}$. Using (17), $\left\|\boldsymbol{\epsilon}_{k}\right\|_{2}$ can be upper bounded in terms of $\left\|\epsilon_{0}\right\|_{2}$ as follows

$\left\|\epsilon_{k}\right\|_{2} \leq \alpha^{k}\left\|\epsilon_{0}\right\|_{2}+\mu\left\|\left[\begin{array}{cc}\mathbf{0} & A^{\top} \\ -A & \mathbf{0}\end{array}\right]\right\|_{2} \sqrt{N+M} \frac{k \alpha^{k-1} d}{2^{R}}$

Thus, $\lim _{k \rightarrow \infty}\left\|\epsilon_{k}\right\|_{2}=0$ which implies that the primal and dual variables under the quantization scheme $\mathcal{Q}_{\mathrm{u}}$ converge to their optimal values.

\section{REFERENCES}

[1] S. Shakkottai and R. Srikant. "Network optimization and control.", Found. Trends Netw., vol. 2, no. 3 pp. 271-379, Jan. 2007.

[2] T. Alpcan and T. Basar, "A Utility-Based Congestion Control Scheme for Internet-Style Networks with Delay," IEEE Trans. Netw., vol. 13, no. 6, pp. 1261-1274, Dec. 2005.

[3] F. P. Kelly, A. Maulloo, and D. Tan, "Rate control for communication networks: Shadow prices, proportional fairness and stability," J. Oper. Res., vol. 49, no. 3, pp. 237252, Mar. 1998.

[4] A. Nedić, A. Olshevsky, A. Ozdaglar and J.N. Tsitsiklis, "Distributed subgradient methods and quantization effects," IEEE Conf. on Decision and Control Conference (CDC), pp.4177,4184, 9-11 Dec. 2008.

[5] M.G. Rabbat and R.D. Nowak, "Quantized incremental algorithms for distributed optimization," IEEE J. Sel. Areas. Comm., vol. 23, no. 4, pp. 798,808, April 2005.

[6] Y. Cui, and V.K.N. Lau, "Convergence-optimal quantizer design of distributed contraction-based iterative algorithms with quantized message passing.", IEEE Trans. Signal Process., vol. 58, no. 10, pp. 5196-5205, Oct. 2010.

[7] D. Yuan, S. Xu, H. Zhao and L. Rong, "Distributed dual averaging method for multi-agent optimization with quantized communication," Systems \& Control Letters, vol. 61, no. 11, pp. 1053-1061, Nov. 2012.

[8] P. Yi and Y. Hong, "Quantized Subgradient Algorithm and Data-Rate Analysis for Distributed Optimization," IEEE Trans. Control Netw. Syst., vol. 1, no. 4, pp. 380-392, Dec. 2014.

[9] T. Alpcan and S. Dey, "An information-theoretic analysis of distributed resource allocation," 52nd IEEE Conf. on Decision and Control, Florence, Italy, Dec. 2013.

[10] P. Minero, M. Franceschetti, S. Dey and G.N. Nair, "Data Rate Theorem for Stabilization Over Time-Varying Feedback Channels," IEEE Trans. Autom. Control, vol. 54, no. 2, pp.243-255, Feb. 2009.

[11] J.S. Freudenberg, R.H. Middleton, V. Solo, "Stabilization and Disturbance Attenuation Over a Gaussian Communication Channel," IEEE Trans. Autom. Control, vol. 55, no. 3, pp.795-799, March 2010.

[12] T. Basar and G. J. Olsder, "Dynamic noncooperative game theory", 2ed ed., New York, Academic Press, 1995.

[13] G.N. Nair and R.J. Evans, "Stabilizability of stochastic linear systems with finite feedback data rates," SIAM J. Control Optim., vol. 43, no. 2, pp. 413-436, 2004.

[14] T. M. Cover and J. A. Thomas, "Elements of Information Theory", New York: Wiley, 2005. 


\section{University Library}

\section{- M M N E R VA A gateway to Melbourne's research publications}

Minerva Access is the Institutional Repository of The University of Melbourne

Author/s:

Nekouei, E;NAIR, G;Alpcan, T

Title:

Convergence Analysis of Quantized Primal-dual Algorithm in Quadratic Network Utility Maximization Problems

Date:

2015

Citation:

Nekouei, E., NAIR, G. \& Alpcan, T. (2015). Convergence Analysis of Quantized Primal-dual Algorithm in Quadratic Network Utility Maximization Problems. Proceedings of the 2015 IEEE 54th Annual Conference on Decision and Control, 54rd IEEE Conference on Decision and Control,CDC 2015, pp.2655-2660. IEEE. https://doi.org/10.1109/CDC.2015.7402616.

Persistent Link:

http://hdl.handle.net/11343/241548 